\title{
KERNFORSCHUNGSZENTRUM
}

\author{
KARLSRUHE
}

SEPTEMBER 1961

KFK 85

INSTITUT FÜR NEUTRONENPHYSIK UND REAKTORTECHNIK

SOME REMARKS ON THE EFFECT OF A NONUNIFORM TEMPERATURE DISTRIBUTION ON THE TEMPERATURE DEPENDENCE OF RESONANCE ABSORPTION

LAWRENCE DRESNER

KERNREAKTOR 


\title{
KERNREAKTOR \\ Bau- und Ectri:is=-Occolicuhaft m. b. H
Verwaltung der Zentralbucherel
}

\section{Some Remarks on the Effect of a Nonuniform Temperature Distribution on the Temperature Dependence of Resonance Absorption}

\author{
LawRence Dresner* \\ Institut für Neutronenphysik und Reaktortechnik, Kernreaktor Bau- und Betriebs-Gesellschaft m.b.H., \\ Karlsruhe, Germany \\ Received November 28, 1960
}

\begin{abstract}
The influence of a non-uniform temperature distribution on the absorption of neutrons in a purely absorbing resonance of an isolated lump is studied. It is shown that in practical situations the absorption in the lump depends only on its average temperature and not on whether its temperature distribution is uniform or not.
\end{abstract}

The criticality, on one hand, and the initial time behavior of heterogeneous reactors following a large instantaneous reactivity addition, on the other, depend to a great extent on the increase in resonance absorption with increasing temperature. Therefore, the temperature dependence of resonance absorption is one of the important quantities determining both the criticality and safety of heterogeneous reactors. This temperature dependence has been the subject of much study in the past, but in all previous work the temperature distribution was uniform in the absorber lump, quite in contrast to the state of affairs in an actual reactor. The effects of non-uniformity in the temperature distribution may be important, and it is the purpose of this paper to discuss them.

We shall only consider the case of absorption in an isolated lump (i.e., no Dancoff effect) by a purely absorbing resonance (i.e., $\Gamma \gamma \approx \Gamma$ ). Furthermore, we shall ignore potential scattering altogether. Thus we shall not consider either monoenergetic scattering or scattering with moderation in the absorber lump. Under these conditions the effective resonance integral of the absorber is given by the equation

$$
\begin{aligned}
& N V^{Y} I \phi=\int_{\mathrm{res}} d u \int_{\mathrm{S}} d S \int_{\mathbf{n} \cdot \boldsymbol{\omega}>0} d \omega \frac{\cos \vartheta}{\pi} \frac{\phi}{4} \\
& \cdot\left(1-\exp \left\{-N \sigma_{0} \int_{0}^{l\left(\mathrm{r}_{s}, \boldsymbol{\omega}\right)} \psi[\theta(s \omega), x] d s\right\}\right)
\end{aligned}
$$

* On leave from Oak Ridge National Laboratory, Oak Ridge, Tennessee operated by Union Carbide Corporation for the U. S. Atomic Energy Commission. where

$$
\begin{aligned}
& N=\text { the atomic density of absorber atoms } \\
& \text { in the lump } \\
& \checkmark=\text { the lump volume } \\
& I \quad=\text { the effective resonance integral } \\
& \phi \quad=\text { the flux in the moderator, taken as uni- } \\
& \text { form in space and lethargy } \\
& u \quad=\text { the lethargy } \\
& d S \quad=\text { the differential element of the lump } \\
& \text { surface, } S \\
& \mathbf{r}_{s} \quad=\text { the position of a point on the lump } \\
& \text { surface } \\
& \mathbf{n}=\text { the inward normal to } S \text { at } \mathbf{r}_{S} \\
& =\mathbf{a} \text { unit vector at } \mathbf{r}_{S} \\
& =\text { the angle between } \omega \text { and } n \\
& =\text { the peak resonance cross section in the }
\end{aligned}
$$


and where $\psi(\theta, x)$ is given by

$$
\psi(\theta, x)={ }_{2 \sqrt{\pi}}^{\theta} \int_{-\infty}^{+\infty} \frac{\exp \left[-\left(\theta^{2}, 4\right)(x-y)^{2}\right]}{1+y^{2}} d y
$$

By using the inecpuality (I)

$$
\int f(v) e^{--1} d v \geqq \exp \left(-\int f(v) v^{\prime} d v\right)
$$

where $f\left(l^{\prime}\right)$ is any normalized probability frequency function, we can rewrite (1) as

$$
\begin{aligned}
N V I \phi & \leqq \frac{N \phi}{4} \int_{\text {ress }} d u\left(1-\exp \left\{-N \sigma_{0} \int_{s} \frac{d S}{S}\right.\right. \\
& \left.\left.\cdot \int_{\mathbf{n} \cdot \omega>0} d \omega \int_{0}^{l\left(r_{s}, \omega\right)} d s \frac{\cos \vartheta}{\pi} \psi\left[\theta(s \omega), v^{l}\right]\right\}\right)
\end{aligned}
$$

As we shall see liter, the difference between the Rils of (I) and the RHS of (t) is actually rather small. Hence, in what follows we shall drop the $<-$ sign. The exponent in (4) ean be rewritten by interehanging the order of the $s$ and $\omega$ integrations:

$$
\begin{aligned}
& \int_{S} \frac{d S}{S} \int_{\mathrm{n} \cdot \omega>0)} d \omega \int_{0}^{l\langle\mathrm{r} S \omega)} d s \frac{\cos \vartheta}{\pi} \psi[\theta(s \omega), k] \\
& =\frac{1}{\pi S} \int d \omega \int_{s} d S \int_{0}^{l\left(r_{N} \omega \omega\right)} d s \\
& \cdot \cos \theta L(\mathrm{n} \cdot \omega) \psi[\theta(s \omega), x]
\end{aligned}
$$

where $l^{\prime}(y)=0$ if $y<0$ and $l^{\circ}(y)=1$ if $y>0$. The last two integrals over $s$ and $s$ give exactly the volume integral of $\psi$ over 1 . Thus finally we have

$$
\begin{aligned}
\frac{1}{\pi \Gamma} \int d \omega \int_{S} d S \int_{0}^{l\left(\boldsymbol{r}_{N}, \omega\right)} d s & \cos \vartheta l(\mathfrak{n} \cdot \omega) \\
\cdot \psi[\theta(s \omega), x] & =l \psi(\theta, x)
\end{aligned}
$$

where the bar over $\psi$ denoten a spatial average over $r$ and $\bar{l}=4 T^{\circ} S$. Substituting $(6)$ into $(4)$ now gives

$$
\begin{aligned}
I & \left.\left.\left.=(N \bar{l})^{-1} \int_{\mathrm{res}} d u\right\}\right]-\exp \left[-N \sigma_{0} \bar{l} \overline{\psi(\theta, x)}\right]\right\} \\
& =(N \bar{l})^{-1} \frac{\Gamma}{E_{0}} \int_{0}^{\infty} d x\left\{1-\exp \left[-N \sigma_{0} \overline{\psi(\overline{\psi(\theta, x)}]\}}\right.\right.
\end{aligned}
$$

In the last step we have used the relation $-d u=d x(\Gamma \cdot 2)$.

The difference between the RHS of $(4)$ and the RHS of (1) due to the use of inequality (3) can be calculated in the ase of no Doppler effect at all, i.e., $\psi=\left(1+x^{2}\right)^{-1}$, by the method of Crurevich and Pomeranchouk (2). These authors find that the ratio of the two RHS's is $\bar{l}^{12} / \bar{l} 12$ where the bars denote averages with resperet to the chord-length distribution

$$
f(l)=\int_{S} \frac{d S}{S} \int_{\mathbf{n} \cdot \omega>0} d \omega \frac{\cos \vartheta}{\pi} \delta\left[l-l\left(\mathbf{r}_{s}, \omega\right)\right]
$$

and where $I$ still also equals $4 \mathrm{~T} / \mathrm{s}$. The factor $\overline{l^{1 / 2}} / 7^{1 / 2}$ is respectively equal to $0.980,0.975$, and 0.943 for spheres, eylinders, and slabs. Thus only a small crror is introduced into (4) when it is taken with the equality sign.

That this last conchusion does not depend very strongly on the precise shape of the line can be reen by repeating the above caleulation for a line with the shape $\left(1+x^{n}\right)^{-1}, n>1$. In this ease, the ratio of the RHS of $(1)$ to that of $(4)$ in griven by $\overline{l^{1 / n}} / \bar{l}^{1}$. Fol spheres and slabs this ratio has a shallow minimum near $n=2$, rising to unity for $n=1$ and $n=x$. The values at the minimum are exsentially those given in the previous paragraph. Thus we expect that for spheres and rylinders especially, the orrol arising from the use of (3) in (1) is neither very great nor varies rapidly with the shape of the line.

According to (2)

$$
\begin{aligned}
& \psi(\theta, x)=\int f(\theta) d \theta \cdot \begin{array}{c}
\theta \\
2 \sqrt{\pi}
\end{array} \\
& \int_{-\infty}^{+\infty} \frac{\exp p\left[-\left(\theta^{2} / 4\right)(x-y)^{2}\right]}{1+y^{2}} d y \\
& =\sum_{2 \sqrt{\pi}}^{\bar{\theta}} \int_{-\infty}^{+\infty} \frac{d y}{1+y^{2}} \int \frac{f(\theta) \theta}{\bar{\theta}} \\
& \cdot \exp \left[-\left(\theta^{2}: 4\right)(x-y)^{2}\right] d \theta
\end{aligned}
$$

where $f\left(\theta^{\prime}\right) d \theta^{\prime}$ is that fraction of the lump rolume in which $\theta$ lies between $\theta^{\prime}$ and $\theta^{\prime}+d \theta^{\prime}$. Now $f(\theta) \bar{\theta}=$ $2 g\left(\theta^{2}\right)$, where the latter function, $g$, is then a posible normalized distribution of $\theta^{2}$. In this case, it follows from $(9)$ and $(3)$ that

$$
\overline{\psi(\theta, x)} \geqq \underset{2 \sqrt{\pi}}{\bar{\theta}} \int_{-\infty}^{+\infty} \exp \left[\frac{\left.-\frac{\left\langle\theta^{2}\right\rangle}{4}(x-y)^{2}\right]}{1+y^{2}} d y\right.
$$

Here the braces denote an average with resperet to $g$. Howerer,

$$
\left\langle\theta^{2}\right\rangle=\int \theta^{2} g\left(\theta^{2}\right) d \theta^{2}=\int \frac{\theta^{3} f(\theta) d \theta}{\bar{\theta}}=\frac{\overline{\theta^{3}}}{\bar{\theta}}
$$

where the bars, as usual, denote space averages.

From (10) and (11) it follows that

$$
\begin{gathered}
\overline{\psi(\theta, x)} \geqq c^{-1} \psi(c \bar{\theta}, x) \\
c^{2}=\bar{\theta}^{3} / \bar{\theta}^{-3}
\end{gathered}
$$


from (12) and (7) it then follows that

$$
I \succsim c^{-1} I_{\text {unlf }}\left(\bar{l} \rightarrow c^{-1} \bar{l}, \quad \theta \rightarrow c \bar{\theta}\right)
$$

whore $I_{\text {unu }}$ is the resonance integral of a lump of the same material and shape, with a size given by $c^{-1} \bar{l}$, and at a aniform temperature $c \bar{\theta}$. The $\sim$ sign oceurs rather than an equality sign to indicate that (13) holds as an inequality only within the (small) error arising from dropping the < sign in (4).

f-Distributions are concesivable in which $c$ does not differ very much from unity, although $\theta$ varies over a reasonably wide range of values. (We shall see an example of such a distribution shortly.) If $c-1$ is small, howerer, the right- and left-hand sides of (12a) ramnot be very different. For, indeed, their integrated difference is giren by

$$
\int\left[\overline{\psi(\theta, x)}-c^{-1} \psi(c \bar{\theta}, x)\right] d x=\frac{c-1}{c} \pi \geqq 0
$$

and since the integrand is positive everywhere, it is rear that if $c-1 \ll 1, \overline{\psi(\theta, x)}$ and $c^{-1} \psi(c \bar{\theta}, x)$ (annot be very different at all. In such a case we would expert that a ralid approximation to (13) would be the equality

$$
I=c^{-1} I_{\mathrm{un} \mathrm{If}}\left(l \rightarrow c^{-1} \bar{l}, \theta \rightarrow c \bar{\theta}\right) \approx I_{\mathrm{un} \mathrm{If}}(\bar{\theta})
$$

where $I_{\text {unf }}(\vec{\theta})$ is the resonanee integral of the actual lump being considered at a uniform temperature $\bar{\theta}$.

In order to pursue this study further and, in particular, test the accuracy of (15), some reasonable f-distribution must be assumed. Iet us therefore imagine that in a relindrical fuel rod in a power reactor the finsion heat souree is effectively spatially uniform. In this cane the temperature distribution in the rod will be parabolic, i.e.,

$$
T(1)=T_{0}+\left(T_{1}-T_{01}\right)(r R)^{2}
$$

where $r$ is the radial coordinate in the rod, $R$ is the radius, and $T, T_{0}$, and $T_{1}$ are, respectively, the temperatures at any point, at the center, and at the boundary. If $T_{1}$ is not rery different from $T_{0}$, the corresponding distribution of $\theta$ is also parabolice, i.e.

$$
\theta(r)=\theta_{0}+\left(\theta_{1}-\theta_{41}\right)(r R)^{2}
$$

From (17) it follows that

$$
\begin{aligned}
& f(\theta)=\left(\theta_{1}-\theta_{0}\right)^{-1} \quad \theta_{0} \leqq \theta \leqq \theta_{1} \\
& q\left(\theta^{2}\right)=\left(\theta_{1}^{2}-\theta_{0}{ }^{2}\right)^{-1} \quad \theta_{11^{2}}^{2} \leqq \theta^{2} \leqq \theta_{1}{ }^{2}
\end{aligned}
$$

i.c., $f$ and $g$ are both uniform distributions. Ife will assume henceforth for the sake of argument that the distributions (18) hold exactly; fortunately, our final conclusions do not depend reritically on the details of these $f$-and $g$-distributions. With respect to general form (18) is, of course, representative.

From (18a) it follows that

$$
c=\left(\frac{\overrightarrow{\theta_{3}}}{\bar{\theta}_{3}}\right)^{1 / 2}=\left[\frac{2\left(\alpha^{2}+1\right)}{(\alpha+1)^{2}}\right]^{1 / 2} ; \alpha=\frac{\theta_{1}}{\theta_{0}}
$$

For $\alpha=\sqrt{2}$, which corresponds to a ratio $T_{0} / T_{1}=2, c$ differs from unity by less than $1.5 \%$; for $\alpha=\sqrt{3 / 2}, c=1.005$. Hence, under the anticipated circumstance that in practical cases $\alpha$ is not too large, we expect (15) to be valid.

We can test this conclusion for the interesting case of extreme 1)oppler broadening (i.e., $\theta \ll 1$ ) for which Wigner et al. (3) have suggested the use of the approximate line shape

$$
\psi(\theta, x)=\frac{\theta \sqrt{\pi}}{2} \exp \left[-\left(\theta^{2} / 4\right) \cdot r^{2}\right]
$$

Equation (20) is particularly appropriate for those resonances which contribute strongly to the temperature roefficient of the resonance integral. From (20) and (18) it follows that

$$
\overline{\psi(\theta, x)}=\sqrt{\pi} \frac{\exp \left[-\left(\theta_{0}^{2}, 4\right) x^{2}\right]-\exp \left[-\left(\theta_{1}{ }^{2}, 4\right) x^{2}\right]}{\left(\theta_{1}-\theta_{0}\right) x^{2}}
$$

Inverting (21) into (7b) and rearranging one obtain

$$
\begin{aligned}
I=\frac{\pi}{2} \sigma_{0} \frac{\Gamma}{E_{i}} \frac{1+\alpha}{\sqrt{\pi} \beta} \int_{0}^{\infty}\left(1-\exp \left\{-\beta \exp \left(-z^{2}\right)\right.\right. \\
\left.\left.\cdot \frac{\left.\left.1-\exp \left[-\left(\alpha^{2}-1\right) z^{2}\right]\right\}\right) d z}{\left(\alpha^{2}-1\right) z^{2}}\right\}\right) d z
\end{aligned}
$$

where $\beta=N \sigma_{0} \bar{l}(\sqrt{\pi} 2) \bar{\theta}$ and $\alpha=\left(\theta_{1} \theta_{0}\right)$. When $\alpha=1, I=I_{\text {unf }}(\bar{\theta})$.

Typical ralue; for the parameters are $X=0.0 .5$ barms ${ }^{-1} \mathrm{c}^{-1}$ for metallic $L, \bar{l}=2 \mathrm{~cm}, \bar{\theta}=0.05$, $\sigma_{11}=5000$ barm. For these values $\beta=22$. Numerieal integration of (2.2) for the four (anes, $\alpha=1, \sqrt{2}$; $\beta=10$, 30 gives the following result: : For $\beta=10$, $I$ and $I_{\text {umf }}(\bar{\theta})$, i.c., $I(\alpha=\sqrt{2})$ and $I(\alpha=1)$, ar' within about $1.5 \%$ of one another, the former being larger. For $\beta=30$, the corresponding difference is about 2.5' $;$. Thus for these conditions our expectattion that (15) would be aceurate is rerified.

Based on the foregoing analysis we are inclined to consider the approximation $I=I_{\text {un } 1 \mathrm{f}}(\bar{\theta})$ as a fairly accurate one ${ }^{1}$. For practical purposes it is virtually

This approximation is originally due to (i. M. Roe "Resonance Absorption of Neutrons in Doppler Broadened Resonances," KAPI,-1241 (October, 1954) Roe assumed that for slabs, $I$ was given by $I_{\text {auf }}\left(\theta_{c}\right)$, where $\theta_{\text {e }}$ was determined by the requirement that the integrals $\int_{0 \psi}^{x} \psi(\theta, x)^{2} d x$ and $\int_{0}^{\infty} \psi^{2}\left(\theta_{t}, x\right) d x$ be the same. This condition gave $\theta$ approxmately equal to $\left.\bar{\theta}-(3 ; 2)\left\{\left(\bar{\theta}^{2}-\bar{\theta}^{2}\right) ; 1+4 \bar{\theta}\right)\right\}$. The form 
identical with the oft-repeated prescription that the actual resonance integral is equal to that of the same lump uniformly held at the average temperature, since the effective temperature determined from $\bar{\theta}$ is usually not very different from the average temperature. The virtue of the present note is that it indicates that this prescription is, in fact, quite accurate, at least in the special case treated.

of the second term depends on how $\theta_{e}$ is determined and is to some extent arbitrary. Thus the real meaning of Roe's result is that if the second term is not too large, $\theta_{e}=\bar{\theta}$ to a good order of approximation.

\section{ACKNow LedgMent}

The author wishes to thank Dr. W. K. Ergen for an interesting correspondence regarding this problem.

\section{REFERENCES}

1. G. H. Hardy, J. E. I.tTthewood, and G. Polya, "Inequalities," p. 137, theorem 187. Cambridge Univ. Press, London and New York, 1934.

2. I. I. Gurevich AND 1. Y. Pomeranchouk, Proc. 1st Intern. Conf. Peaceful Uses Atomic Energy, Geneva 5, 466 (1955).

3. E. P. Wigner, E. Creutz, H. Jupnik, and T. Snyder, J. Appl. Phys. 26, 260 (1955). 